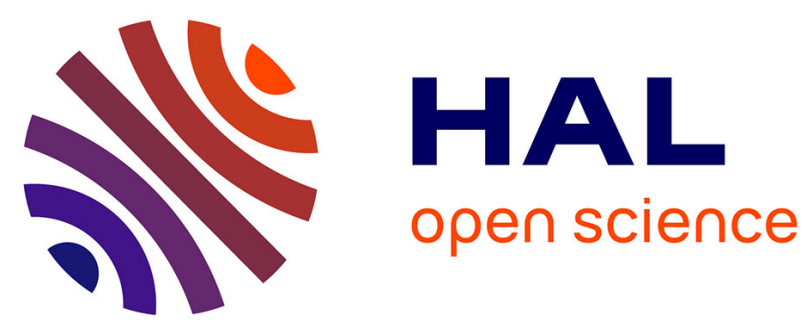

\title{
Le sanctuaire celto-romain du Mesnil de Baron-sur-Odon (Calvados)
}

Dominique Bertin

\section{To cite this version:}

Dominique Bertin. Le sanctuaire celto-romain du Mesnil de Baron-sur-Odon (Calvados). Gallia - Fouilles et monuments archéologiques en France métropolitaine, 1977, 35 (1), pp.75-88. 10.3406/galia.1977.1556 . hal-01939328

\section{HAL Id: hal-01939328 \\ https://hal.science/hal-01939328}

Submitted on 14 Jun 2020

HAL is a multi-disciplinary open access archive for the deposit and dissemination of scientific research documents, whether they are published or not. The documents may come from teaching and research institutions in France or abroad, or from public or private research centers.
L'archive ouverte pluridisciplinaire HAL, est destinée au dépôt et à la diffusion de documents scientifiques de niveau recherche, publiés ou non, émanant des établissements d'enseignement et de recherche français ou étrangers, des laboratoires publics ou privés.

\section{(이) $\$$}

Distributed under a Creative Commons Attribution - NonCommercial - NoDerivatives| 4.0 


\title{
LE SANCTUAIRE CELTO-ROMAIN DU MESNIL DE BARON-SUR-ODON (Calvados)
}

\author{
par Dominique BERTIN
}

Le monument gallo-romain du llesnil, lieu-dit de la commune de Baron-sur-Odon (Calvarlos), est un sanctuaire de tradition reltique, situé tout près du point le plus haut de la plaine de Caen, la rote 11 , a $10 \mathrm{~km}$ au sud-ouest de la ville à proximité d'un carrefour de vieux chemins, une voie romaine dite Chemin Haussé ou Chemin Guillaume le Conquérant, et un chemin creux, encore profond de $3 \mathrm{~m}$, dit Chemin Gaulois (fig. 1). En plein corur de lat cité antique des Viducasses, il est relié par le Cihemin Haussé a la capitale, Vieux, l'antique Araegenuar ou dregenue, dont il n'est eloigné que de $3 \mathrm{~km}$ à vol d'oiseau. Les vestiges, repérés en 19.2 parr un cultivateur en raison des nombreux moellons, tuiles, tessons ef coquilles d'huitres que la charrue amenait à la surface du champ laboure, ont été sommairement fouillés de 1952 à 1956. et un plan des structures fut alors relevé. Depuis cing ans, nous nous sommes proposé, non de reprendre dans son ensemble la fouille de ce grand monument (42 $\mathrm{m}$ de plus grand diametre), mais de donner une stratigraphie du site, d'étudier les structures dans leurs états successifs, de compléter et de corriger le plan déjà dressé, d'établir enfin une chronologie relative en multipliant les sonilages en divers points du site.

Outre le monument proprement dit, nous avons été amené à fouiller une structure, située à $100 \mathrm{~m}$ dans l'axe de l'entrée, qui était ronstituée d'une vaste plate-forme faite d'un dallage épais, lequel recourrait une fosse remplie de pierres calcaires (fig. 2). Celle-ci avait exactement la forme et l'aspect d'une sépulture, mais elle était vide, d'où le nom de "sépulture symbolique» que nous lui avons donnée. Ciette découverte est peut-être la plus intéressinte, car elle pose à nouveau le problème des rapports des lieux-dits, peut-être hàtivement, de culte funéraire aver les sanctuaires dans la Gaule romaine. Enfin, à proximite de la fosse, mais sans rapport aver elle, nous avons trouvé un habitat de la Tène finale. Cette dernière découverte confirme l'existence d'un sanctuaire indigène antérieur au monument gallo-romain reconnu, hỵpothèse que la fouille du monument lui-mème arait déjà permis de formuler. Ajoutons qu'un fourreau d'épée celtique à décor incrusté d'or figurant des dragons affrontés, et une libule de l'époque du gallo-romain précoce à ressort

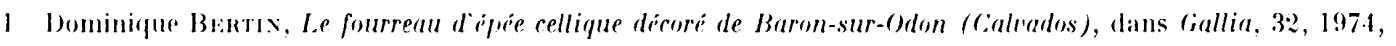
p. 243-2.18, 5 fig. 

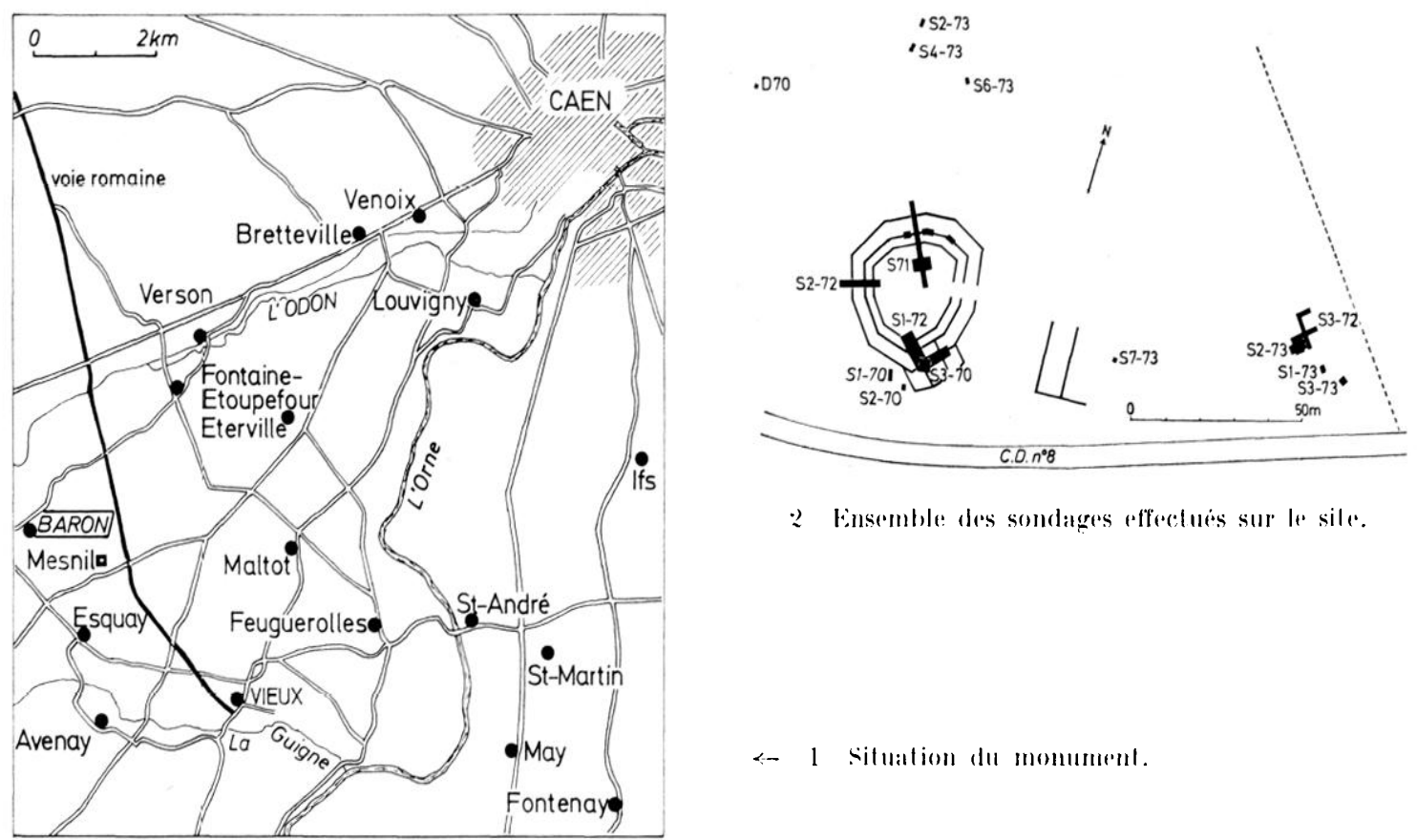

2 Ensemble des somdages effochues sur le sile.
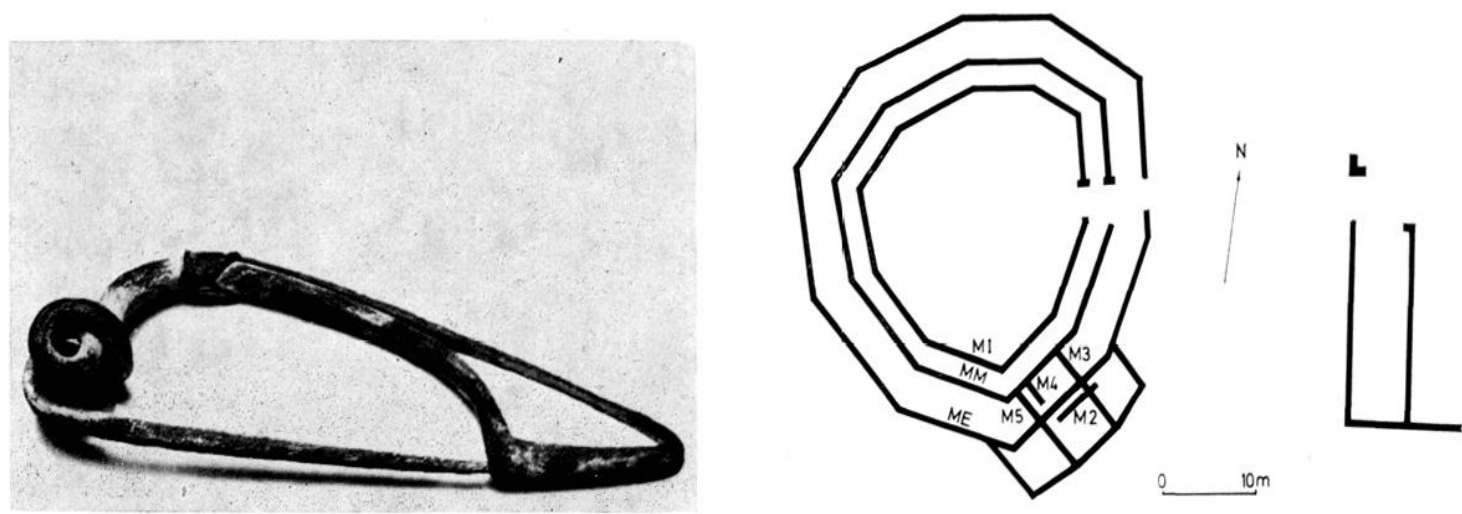

3 Fibule de l'époque dı gallo-romain pricoce.

4 Plan d'ensemble du momument.

nu et à corde intérieure à l’are (fig. 3)² ont été découverts sur le site, mais malheureusement hors stratigraphie.

Le sanctuaire est implanté non pas au point le plus haut de la plaine, mais légèrement en contre-bas, à la naissance d'un vallon, en bordure d'une vallée sèche, en un point où l'on est vu de très loin et d'où l'on découvre largement le paysage. Le monument est constitué de trois enceintes décagonales concentriques, délimitant deux galeries qui enserrent une aire centrale à ciel ouvert (fig. 4). Le décagone est irrégulier et allongé dans le sens nord-sud/estouest, ses côtés ont $14 \mathrm{~m}$ de long en moyenne, et l'entrée du monument est située exactement à l'est. La cour intéricure a un diamètre moyen de $26 \mathrm{~m}$, l'enceinte extérieure de $42 \mathrm{~m}$. Les

2 Locien LrRat, Calalogue des colleclions archeologiques de Besançon $I J$. Les fibules gallo-romaines Annales lilleraires de l'liniversili de Besancon, 1. III. fasc. 1, 1956, p. I, fir. I . 
galeries interieure et extérieure sont larges respectivement de $2,10 \mathrm{~m}$ at de $3,60 \mathrm{~m}$; seules parties couvertes dans le sanctuaire, elles ont recu une toiture de tuiles qui était sans doute portée par une colonnarle de bois ; toutefois, un fragment de colonne de pierre a été découvert près de l'entrée.

Deux caractéristiques originales : la forme décagonale et l'absence de cella. Si le polygone, et en particulier l'octogone, est une forme assez fréquemment rencontrée dans les temples celto-romains (sanctuaires de Morilasgus à Alésia, de Champallement et de Saint-Révérien dans la Nièvere, de Mayence, par exemple), le décagone est unique, à notre connaissance. L'absence de cella n'est pas inexplicable dans ce sanctuaire de hauteur très marqué par la tradition indigène : les (iaulois sculptaient peu, figuraient rarement leurs dieux dans la pierre du moins sous l'Indépendance - et n'avaient done pas besoin de Lemple au sens de "maison du dieu». Les premiers sanctuaires ont ete parfois de simples enceintes enserrant le lieu sacré, résidence du dieu topique : a Friesen dans le Haut-Rhin, aux Fontaines-salées dans l'Yonne, les sanctuaires du a siècle sont de plan circulaire sans cella, centrés dans le premier cas autour d'un puits, dans le second autour d'un bassin, et munis d'une enceinte et d'un promenoir périphérique. Le déambulatoire semble aroir joué un grand rôle dans le rituel celtique : il permettait peut-être les processions autour du point sacré qui était censé ètre la résidence du dieu, ou autour de la cella. Selon Posidonios

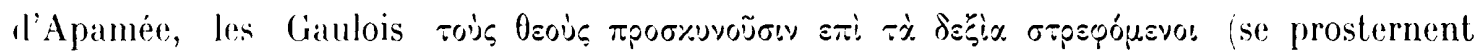
devant leurs dieux en se tournant (de la gauche) vers la droite). S'il n'y a pas de cella à Baron, le déambulatoire semble avoir une importance particulière car il est double : il y a deux galeries périphériques. Autour de quoi le sanctuaire de Baron était-il centré? Les fouilleurs de 1954 ont trouvé, au centre exact du monument, une cavité d'environ $3 \mathrm{~m}$ de diametre et de $1 \mathrm{~m}$ de profondeur, remplie de terre noire mais vide de tout mobilier. La zone centrale du sanctuaire ayant été très bouleversée, il a été impossible de vérifier ces données et aucune interprétation de cette cavité ne saurait ètre proposée avec certitude. Tout au plus peut-on supposer l'existence d'un mundus ou d'un loculus central, dont la signification nous échappe. Quant à la forme polygonale du temple, ce n'est, à notre avis qu'un dérivé de la forme circulaire, commandé ici par la forte dénivellation du terrain du nord vers le sud. Au reste, il est difficile de proposer une antériorité de la forme carrée par rapport à la forme circulaire. Cne étude plus générale montre que ces deux types de forme apparaissent en mème templs, la forme carrée étant cependant plutòt réservée aux petites cellae (Saint-Germain-le-Rocheux, Còte-d'()r, par exemple), et la forme circulaire aux grandes enceintes (Mandeure, Ioubs, ou Saint-Maur-en-Chaussée, Oise).

Le monument, malgré ses particularités, présente les traits fondamentaux du sanctuaire celtique, à l'exception de la cella : position élevée mais à flanc de côteau, péribole clos delimitant de terroir sacre du terroir profane, galeries pour le rite de la déambulation, orientation st. Lat divinite reste inconnue, mais on peut supposer soit une divinité de hauteur, topique, soit une divinite tribale, le temple étant situé au roxur de la cité viducasse, près du chef-liesu. Linfin, le sanctuaire est accompagné d'annexes, dont un long bàtiment rectangulaire au surl-est et diverses constructions en rapport plus ou moins direct avec le temple, réparties sur plusieurs hectares antour du monument. Si le sanctuaire a connu un 


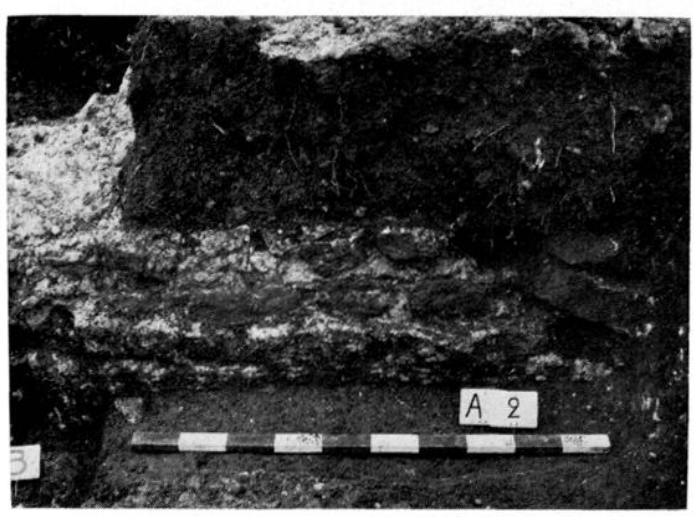

5) Mur de galterie médian M. M.

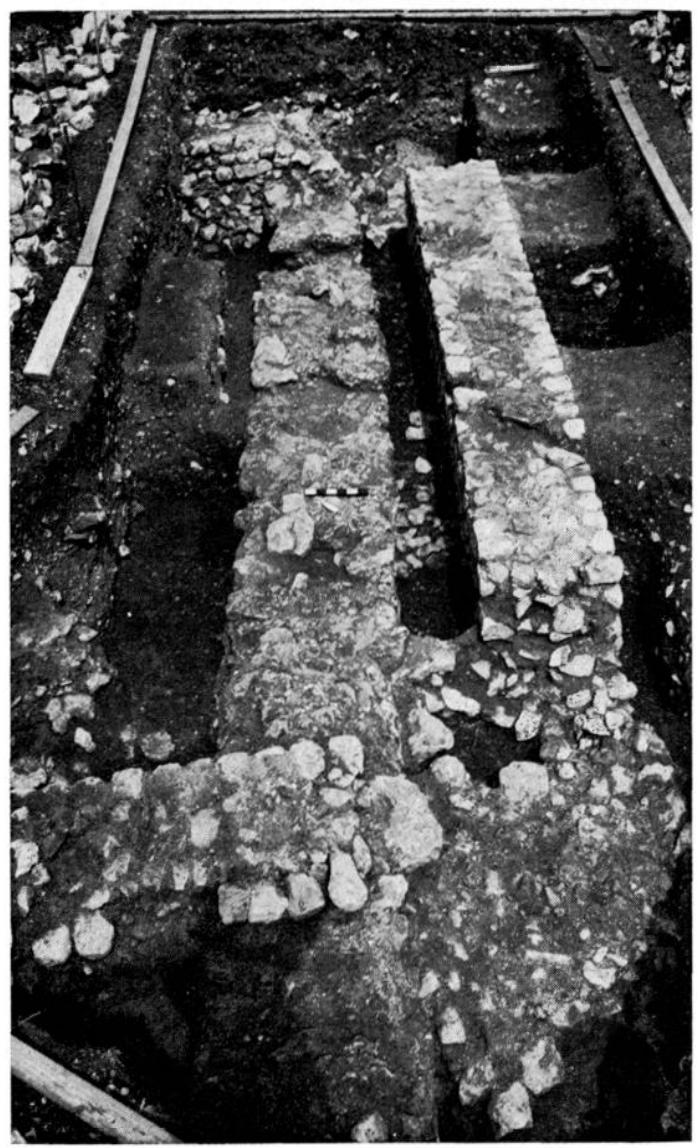

7 Petites pirces annexes an suld du momument

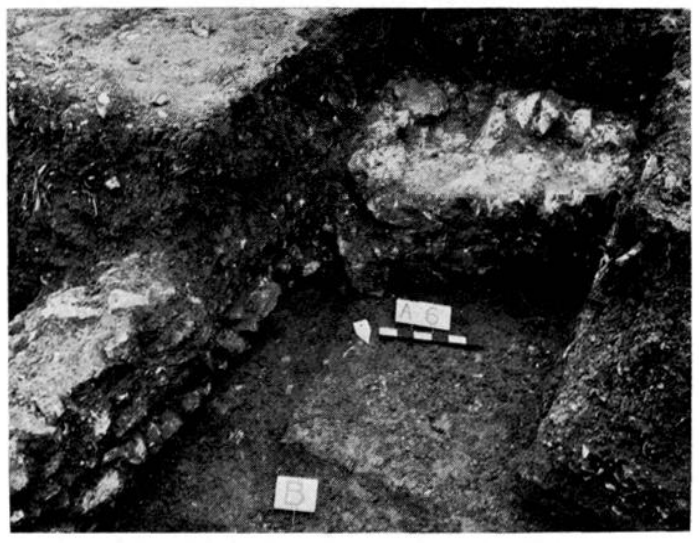

(i) Mur de galerio interieur $\mathrm{MI}$.

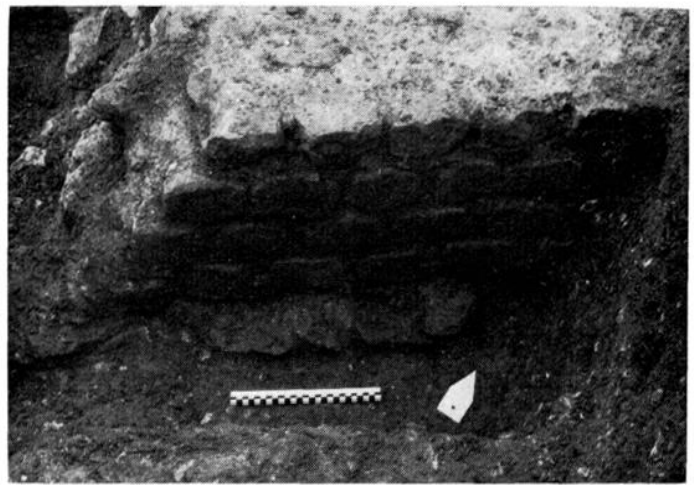

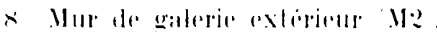

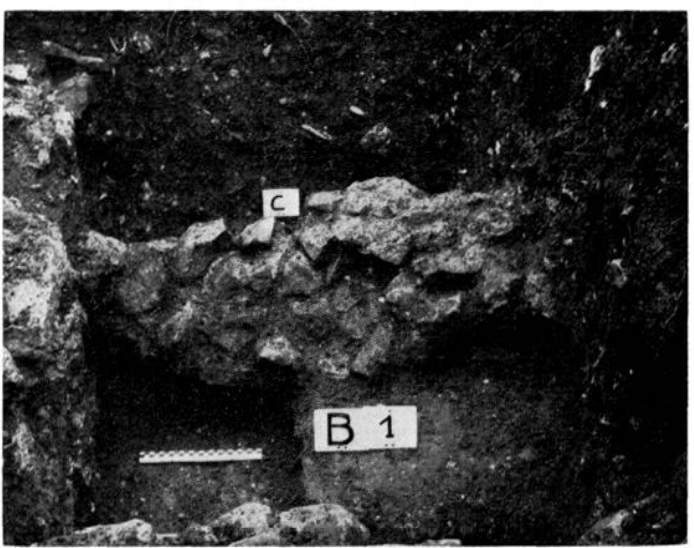

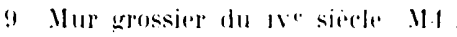

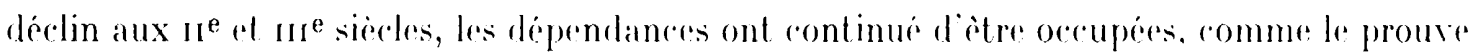
la fouille d'un dépotoir en rapport aver une annexe, et qui a fourni un abondant mobilier céramique du milieu du $\mathrm{I}^{\mathrm{e}}$ siècle. dont un vase sigillé Drag. 37 de Bulrio.

Les structures el la straligraphie ont été étudiées au nord, au sud et à l'ouest. La partie est. Ires bouleversée par les fouilles anciennes, n'a pu etre rrexamine. bu point de ve des structures, 
on a reconnu segt mur- dont trois principaux : le mur de galerie médian (MII), te mur de galerie intérieur (XI), le mur de caleric extérieur (VE). I rois murs apparlenant à une construction poste-

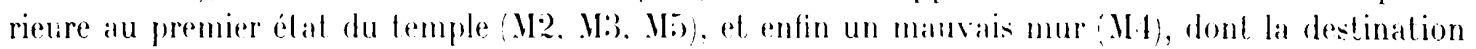
reste inexpliquée.

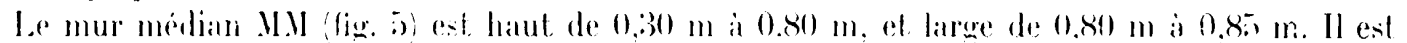
constitur d'un hlocage rentral parementé au suil el a l'onest en petit appareil allongé. et au nord en dalles posees a la vertiale. Il contient des materianx de remploi, ef hien quetant, a notre avis, le plus ancien mur du sanchuare const ruit en dur. asel deja une reconstrurlion.

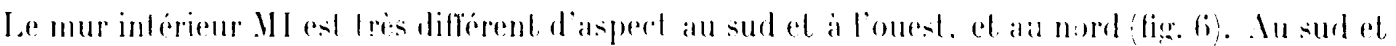

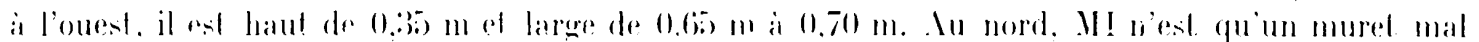

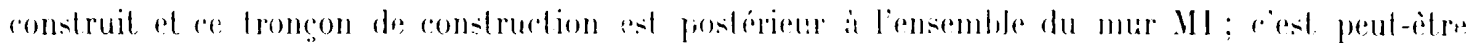
une réfection.

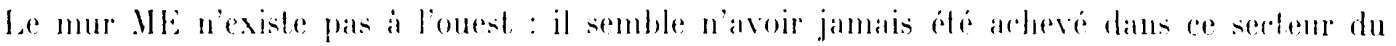

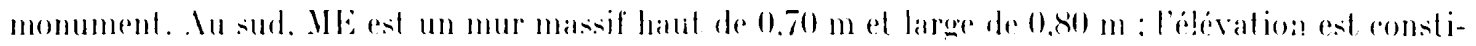

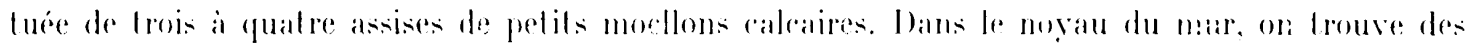

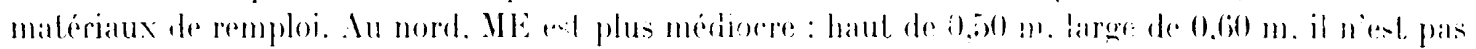

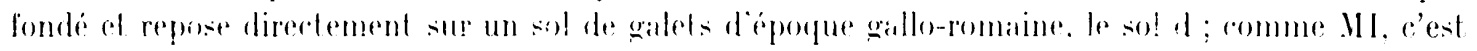

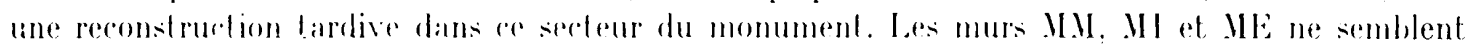

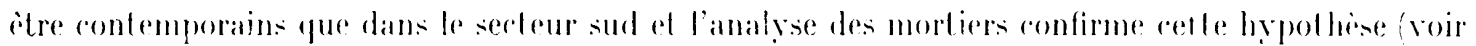
plus has ).

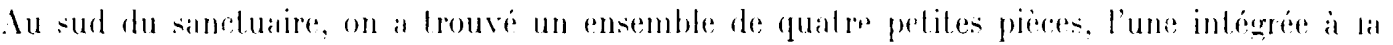

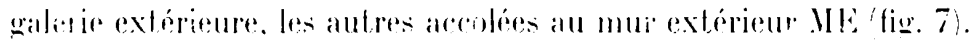

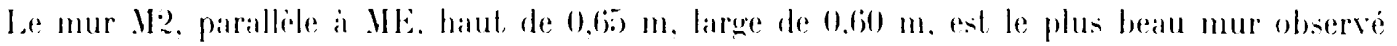
dans le monument (fig. 8). Il est paremente en élevation aver un pelit appareil tres régulier, et les mocellons du parement, biseautés intérieurement. senfoncent profomdément dans le blocage du mur. La face extérieure, destiné a ibre vue, a de soigneusement ravalee, alors que la face interieure devait blre recouverte d'enduits peints. Par ailleurs. les fondations sont maconnées avec un mortier jaunatre grossier. alors que les assises superieures le sonl avec un mortier blane de meilleure qualité ; on verra, en etudiant les murs 193 el Vis, que le nivean de changement de mortier correspond au niveau d'un sol.

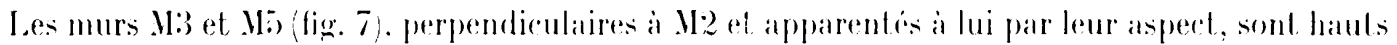

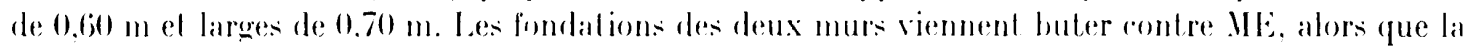
partie en élevation passe au-dessus ; lll: semble donc avoir ete arase pour permeltre la construclion

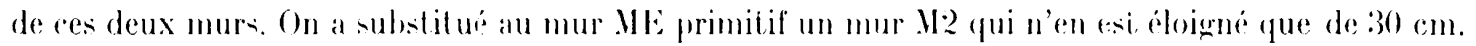
M2, M3 et Mis, de mortier identique, de mode de construction fres prorhe, sont contemporains et ont servi a la construction d'une salle encastrée dans la galerie exterieare du sanctuaire. Dess lors. cette galerie étaif désalfectée au profit de la galerie inlérieure qui al ail la seule a permettre encore les processions. Enfin. le niveau bas de la partie en élevation des murs ll:3 et Vó coüncide exactement

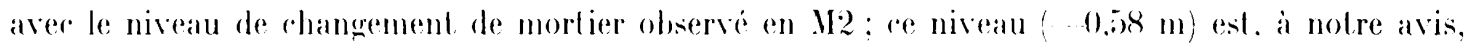
celui du sol a l'intérieur de la salle. Il y a alors une denivellation de 32 am entre le niveau du sol

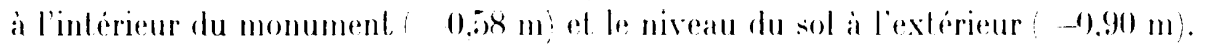

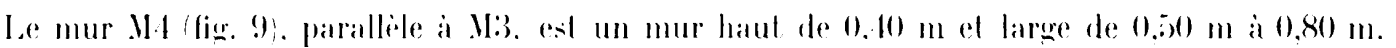

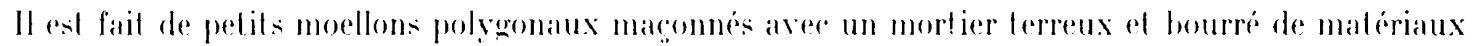
de remploi.sa position est aberrante. el il releve sans doule d une réfertion lardive el d'une réocrupalion du monument, peut-ibe a une fin profane.

Enfin. les deux angles sud de la salle encastrée dans la galerie extérieure du sancluaire ont

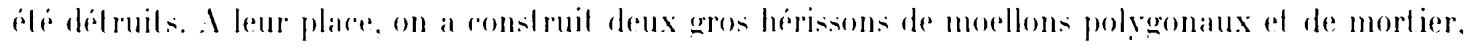

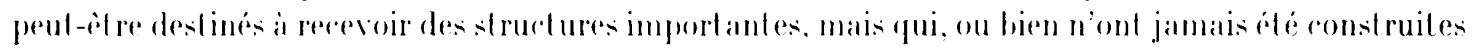
ou bien construiles en pierre de taille. ont éte folalement demantelés par les rarriers mérovingiens pour la récupération de la pierre. 


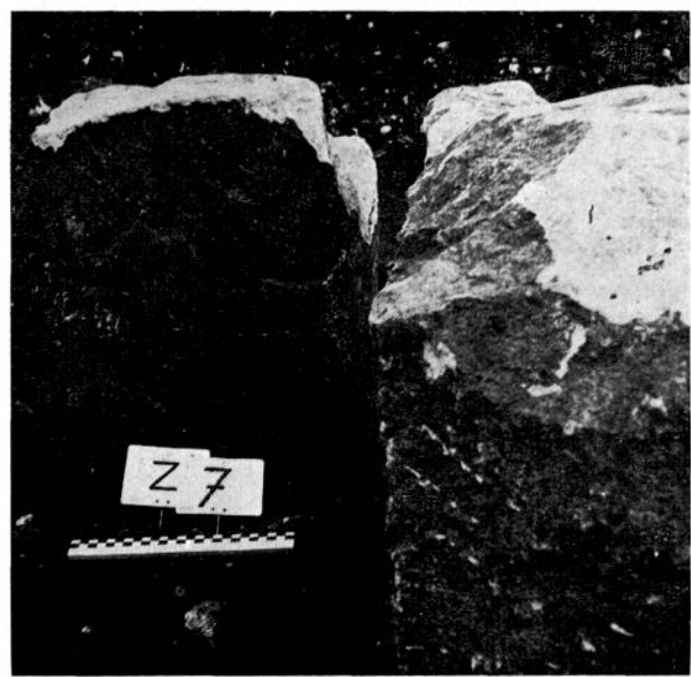

10 Blocs calcaires à goujons.

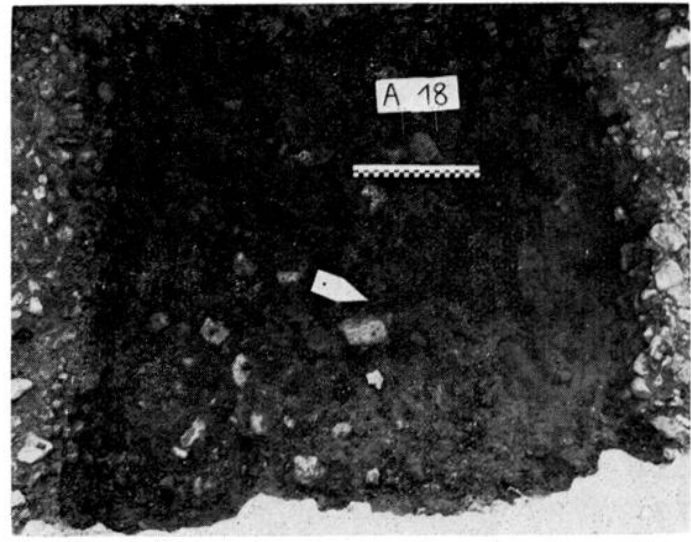

11 Sol gallo-romain de galets roules of dames.

Elude des morliers. Ils ont ete eturlies dans les laboratoires du liRAll de Canen, parr Whe C. Ciubert et par .l. W. Iufournier. Ce sont, dans l'ensemble, des mortiers gris-blane a jaunâtre, de consistance et de dureté moyennes, a l'exerption de celui du mur VIt, tres médiocre. Au sud du temple, les mortiers des murs . VI, ME at M.M présentent des pourcentages identiques en carbonate de calcium, en sable et en argile : environ $20 \%$, de calcium, $65 \%$ de sable et $15 \%$ d'argile. Ces trois murs semblent donc bien etre contemporains, et

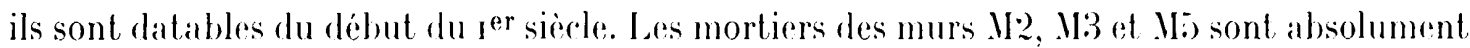
identiques : ce sont des mortiers blanes, durs et compacts, à granulométrie fine. 1ls correspondent à une phase de reconstruction du sanctuaire que l'on peut placer à la fin du ${ }^{\text {er }}$ siècle ou au début du $11^{\mathrm{e}}$ siècle. Enfin, le mortier du mur VIt, très jaune, contient beaucoup d'argile et de calcaire : W4 est une reconstruction grossière, datable du re sièrle, voire du ve siècle.

La stratigraphie. Elle présente cinq niveaux : trois sont gallo-romains, b, c ${ }_{1},{ }_{2}$, l'un est galloromain précoce, $d_{1}$, le dernier est pré-romain, $d_{2}$. Sous une couche de terre arable a, épaisse de $10,25 \mathrm{~m}$, on rencontre une couche de remblai b, épaisse de $0.30 \mathrm{~m}$ i l'intérieur du monument el de (1.5) $\mathrm{m}$ à l'extérieur. Le remblai est plus dense dans les galeries en raison de la proximité des murs arasés. La couche est constituce de matériaux de construction brisés, tuiles, briques, moellons, mortier, fragments de peintures murales unies blanches, jaunes ou rouges. Le mobilier céramique et métallique est rare et remanié. La couche b est un remblai d'effondrement Lardif, du milieu du re siecle.

La couche d'ocrupation archéologique gallo-romaine a áte subdivisée en deux niveaux tris différents, $c_{1}$ et c c. Le niveau c a livé des monnaies constantiniennes el des fragments de pierres sculptées - modillons el corniches - trés brisés. Jeux gros blocs de pierre, dans ce niveau, haut. de $0,48 \mathrm{~m}$, larges de $0,5,5 \mathrm{~m}$ et longs respectivement de $0,70 \mathrm{~m}$ et de $1,10 \mathrm{~m}$. présentant le premier un goujon horizontal, le second un goujon vertical. ont protégé la stratigraphie (fig. 10). Ces blocs et les pierres sculptées qui les arcompagnent rorrespondent a une destruction du monument au milieu du ree siècle, peut-ètre par les chrétiens, car les sculptures semblent avoir íté intentionnellement brisées. Ils proviennent soil de l'entrée, qui aurait été restaurée et agrémentée de sculptures à la fin du ${ }^{\text {er }}$ siècle ou au début du ${ }_{1}{ }^{\mathrm{e}}$ sjeccle, soit d'une cella légèrement excentrée vers le nord-est, 


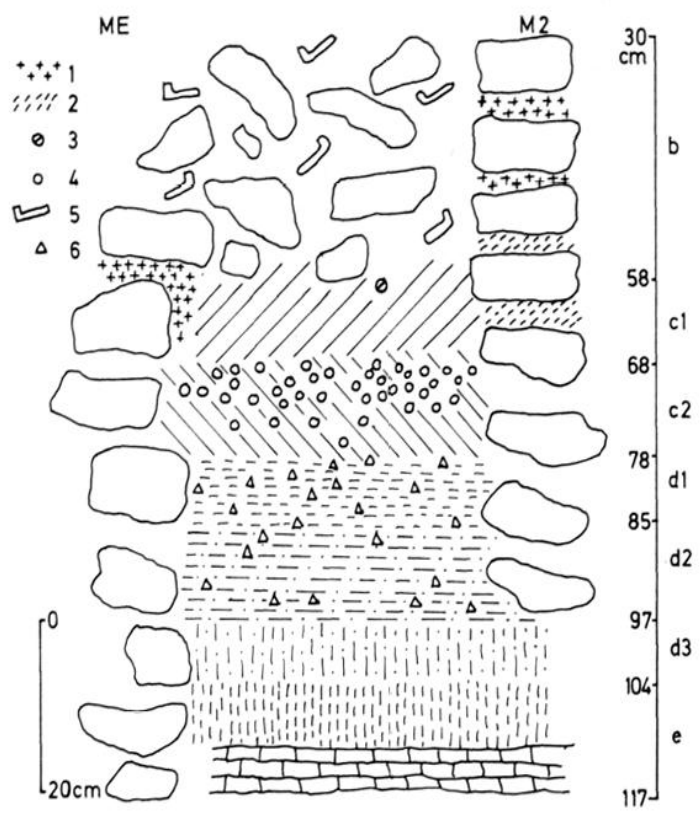

1: loupe stmatigraphique pratiquede sur Ir monument au niveau de la favissa: 1. mortier blane; 2 , mortier jaune; 3 , monnaje; 4 , anneaux; 5 , legula $; 6$, ceramique; - b, I e s.; c 1 , III IVe $^{\mathrm{e}}$; c 2 ,

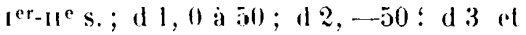
e, couches nalurelles.

qui aurait été complitement démantelée, el dont nous n’avons rien retrouvé. Le niveau $c_{2}$ sous-jacent est un niveau archéologique de la deuxiòme moitié du rer siécle et du début du ne siécle contenant cependant quelques éléments céramique pré-romains qui proviennent des couches sous-jacentes remaniées. Par endroits, les niveaux $c_{1}$ et $c_{2}$ sont remplacés par un sol (sold) de galets de quartz et de silex roulés et à bords vifs, usés et tassés par un fort piétinement (fig. 11). Entre les galets du sol d s'est infiltré, sous l'effet du piétinement, un petit mobilier gallo-romain (anneaux de bronze, clous de fer. tessons de céramique commune, petits bronzes de Tétricus). Nous expliquons la formation du sol d par l'utilisation des galets naturels du site, et par une recharge en galets pris sans doute a la cote 112 où ils abondent -- des alluvions tertiaires aflleurant en cet endroit - afin d'assainir le sol des galeries et de l'esplanade qui entourait le monument. Par ailleurs, au nord du monument, le sol de la galerie extérieure est plus haut de $15 \mathrm{~cm}$ que celui de la galerie intérieure, alors qu'au sud, le sol de la galerie extérieure est plus has de $32 \mathrm{~cm}$ que celui de la galerie intérieure. Le glissement des terres du norl au sud explique ces dénivellations successives, et le pendage naturel du terrain explique aussi pourquoi les murs sont plus profondément fondés au sud qu'au nord.

La couche $c_{2}$ prend enfin un aspect particulier entre les murs M2 et ME au sud du monument: sous la couche d'effondrement b, qui affecte ici un pendage sud-nord, une sorte de cachette aménagée entre les deux murs parallèles recélait 15) anneaux de bronze caractéristiques du site de Baron, comme on le verra plus bas. Nous sommes certainement en présence d'une favissa comparable à celles des fana de Crain, Yonne, et du Portus, saòne-et-I coire : on a profité de la construction de M2 pour se débarrasser des anneaux qui encombraient, le sanctuaire à la fin du rer siècle (fig. 12 et 13).

En raison du hiatus observé entre les niveaux $c_{1}$ et $c_{2}$, il faut admettre qu'il y a eu rupture dans l'occupation du sanctuaire (mais non des dépendances), entre le début ou le milieu du e $^{\mathrm{e}}$ siècle, époque dabandon du monument, et le début du we siècle, époque de. reconstruction du monument rapidement suivie de sa ruine.

La caracteristique des couches sous-jacentes a la couche cest que l'on n'y rencontre aucun élément pouvant se rapporter à l'époque romaine proprement dile. Ces couches sont limitèes au

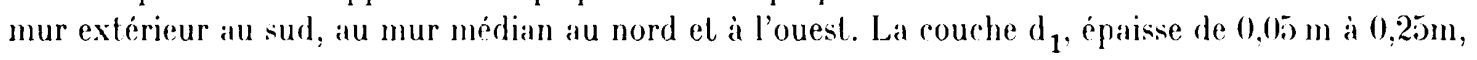




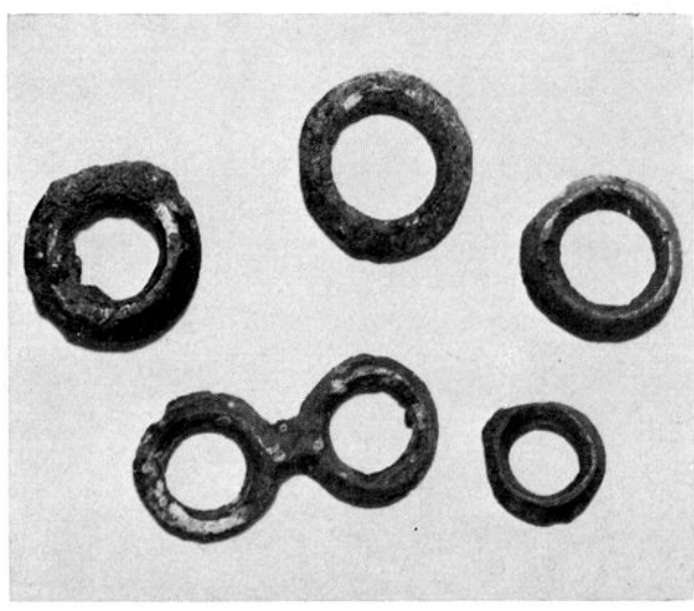

13 Anneaux de la farissst.

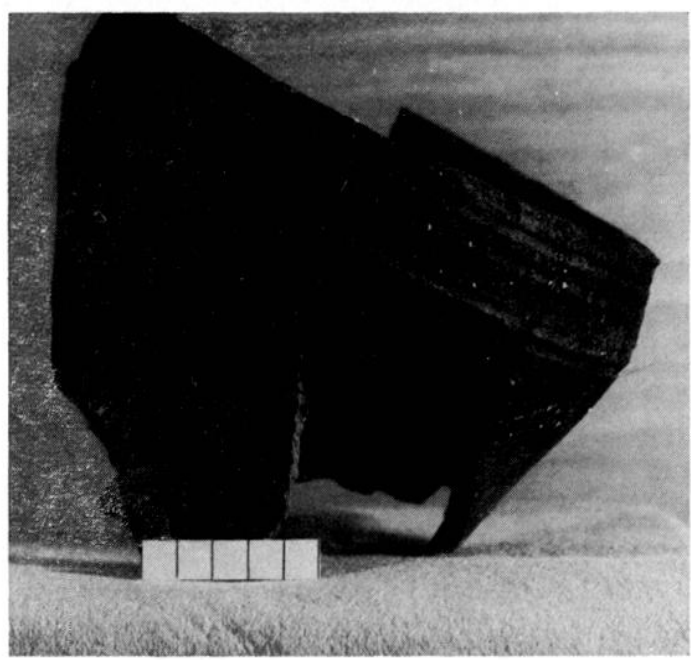

It Vase peigno de Ia Tine finale.

est une couche d'argile noire qui doil sa couleur sombre à la décomposilion de matieres orqaniques. Elle a livré de nombreux os de faune (porcins). des framents d'argile de clayonnage. quelques objets mélalliques, une abondante céramique indigine ef un peu de céramique dile gallo-belge. a pâte claire el à engobe noir. C'est un niveau d'ocrupation de la premiere moilié du der sircle. de l'éporque du gallo-romain précoce. I a courhe $d_{2}$, épaisse de $0.07 \mathrm{~m}$ a $0.10 \mathrm{~m}$. est constituée de galets et d'argile noire. Le mobilier de $d_{2}$ est proche du mobilier de $d_{1}$; cependant, la réramique est exclusivement indigène, et dans l'ensemble plus grossière que celle de $d_{1}$. La couche $d_{2}$ est une couche de La Tene III. I) point de vue des structures, on a seulemenl trouvé a l'inlérieur du monument deux trous de poteaux reusés dans la rouche $d_{2}$ et les couches $d_{1}$ el $d_{2}$ sont des couches d'occupation archéologriques, sans que l'on puisse les qualifier de courhes d'hahital. au moins a l'emplacement. précis du sanctuaire.

Sous la rouche $\mathrm{d}_{2}$, il n'y a pas de paléosol mais une couche d'argile jaune ef de silex $\mathrm{d}_{3}$. puis une couche d'argile jaune e de décomposition du calcaire. Il est insalisfaisant pour l'espril que le paléosol de Baron ait été constitué par une couche de galets tertiaires non recouverts d'humus, mais il est possible que. la couche d'humus étant mince en raison de l'action des vents, du ravinement, et. du pendage du terrain, les premiers habitants du site l'aient piétinée en provoquant ainsi l'infiltration du mobilier qu'ils apportaient : le paléosol se serait alors confondu avec la couche archéologique la plus ancienne. M. J.-L. Ballais, assistant de géomorphologie à l'l'niversite de Gaen, a bien voulu étudier le site. Ia succession des couches, de bas en haut est la suivinle : calcaire. argile jaune, galets et argile noire, argile noire. sol brun, remblai. Le calcaire blanc. bajocien el oolithique, contient des silex. L'argile jaune qui le surmonte et qui contient aussi des silex est issue de la décalcification du substratum calcaire, lors de son érosion au début du Tertiaire. Le lit de galets roulés comporte surtout des quartzites roulés et épandus sur la surface prépliorene probablement au Miorene. Au sein de celte formation, les pelits silex brun-jaune tranchent par leur contour anguleux. L'argile noire, le sol brun et le remblai sont des rouches archéologiques.

Le mobilier. Dans les couches romaines, on a trouves principalement, outre des monnaies. environ 400 petils anneaux de bronze. Ces anneaux. caractéristiques du sile. sont votifs, comme au sanctuaire du Chatelard des Lardiers. Alpes-Maritimes. ou de Crarouville. Eure. On est en droit de penser que des milliers d'anneaux ont été déposés la. I’un diametre extérieur de 1,7 am. d'un diametre intérieur de $1 \mathrm{~cm}$, d'une épaisseur de $0,3 \mathrm{~cm}$ ils présentent une arete vive dans le plan horizontal. Ils ont été coulés dans des moules en série, au moins par deux, car tous présentent la 


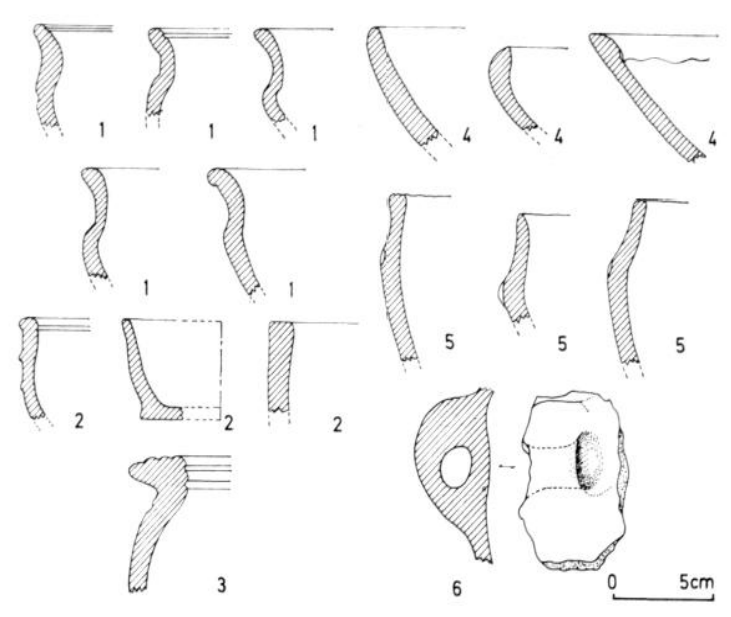

15 Formes de ceramiques de la Time finale : 1 , jattes a profil en $S ;-\ldots$, grobelets a bord droit; - 3, rebord d'olla a cannelures et à gorges internes; -.. 4, ecuelles à bord rentrant ; ... 5, pots légerement carenes avec impressions sur lépaulement; - 6, anse perforé horizontalement.

trace d'une cassure faite avec un burin : les anneaux doubles ou triples sont des anneaux que l'on a oublié de séparer. Ils ont été analysés dans les laboratoires du CRAM de Caen par Mlle (i. Pigreat. Ce sont soit des bronzes au plomb, avec le zinc et le nickel comme impuretés, soit des bronzes au zinc, avec le plomb et le nickel comme impuretés. Cne douzaine d'anneaux d'un plus grand diamètre sont ornés de stries. La couche gallo-romaine précoce a livré deux monnaies gauloises inédites postérieures à la conquête, témoignant d'un art régional très dégénéré el certainement frappées dans: la régrion de leur découverte, d'aprés le I)r J.-B. Colbert de Beaulieu ${ }^{3}$. Ces monnaies figurent au droit une tête de Pallas casquée avec le casque corinthien, tournée à gauche, très stylisée. et au revers un cheval au galop, à droite, à crinière non perlée, conduit par un aurige dont le bras est remplacé par un rinceau en forme d's, aux volutes centrées d'un point.

La couche pré-romaine a livé environ 200 tessons de céramique indigène fort intéressante. Leur pâte va de la teinte cuir au noir. Le dégraissant est le plus souvent siliceux ou calcaire, parfois accompagné de limonite, de glauconie, et de mica blanc ou noir. Les formes indiquent des gobelets, des pots et des jattes, a profil en s, à épaule carénée, à rebord éversé, à fond plat. Ies décors sont asse grossiers et composés de grorges internes sur les rebords parfois ornées d'incisions. d'impressions faites au doigt ou a l'ongle, d'incisions dues à un objet dur, d'ondes faites au peigne. La finition a été réalisée par lissage, polissage ou peignagre. Jans son ensemble. c'est une céramique utilitaire, rarement de luxe, de La T'one finale (fig. 14 et 15)). Cette céramique est comparable à celle qui a été découverte dans les camps fortifiés du Nord-Ouest de la Gaule, fouillés par sir Mortimer Wheeler ${ }^{4}$, à celle d'Aulnat-Sud ${ }^{5}$ et de l'oppidum des Còtes (Puy-de-Dôme) ${ }^{6}$, et à celle de Villers-sur-Mer (Calvados $)^{7}$. Elle se rattache assez bien au noyau céramique armoricain, mais elle est dans l'ensemble moins élaborée et de caractìre plus domestique que la poterie des souterrains-refuges bretons ${ }^{8}$.

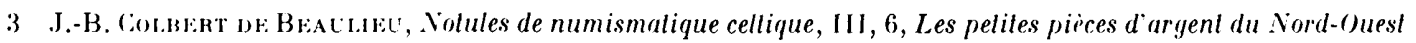
de la tiaule, dans Ogam, VI, 1954, 3, p. 119-130, pl. V, 3, Trailé de numismalique cellique, 1 Méthodologie des ensembles, Paris, 1973 , p. 307, La circulation locale postérieure à 58.

4 Sir Mortmer Whemer, K. M. Ririnardson, Hill-Forls of Norlhern France, Oxford, 1957.

5 J.-J. Hatт, Découverle d'un village gaulois de La Tène III au terroir de Fonlvieille, sur l'emplacement de la base airienne d'Aulnal-Sud, Puy-de-Dôme, dans Bulletin historique et scientifique de l'Auvergne, 62, 1942, p. 36-45, pl. II, III, IV et fig. 2.

6 P. Fychant, Loppidum des Cötes, Clermont-Ferrand (Puy-de-IOome), dans Cellicum, III, suppl, à Ogam. 1962, p. $6 x-76$, pl. $21-25$.

7 R. Camban, E. Lagiat., I he slation de La Tëne finale à Villers-sur-Yer (Calvados), dans Annales de Tormandie, n'2, juin 1964, p. 83-102, pl. 1 à 17.

8 P.-R. Grot, C.-T. Leroux, Y. Oxxer, Le souterrain de Bellevue en Plouegat-Mrysan (Finistere), Laboratoire d'. Inthropologie Préhistorique de la Faculté des Sciences de Rennes, 1967-1968, pl. 1-63. 
L'étude des rapports entre la stratigraphie et les structures a permis d'établir une chronologie relative et une chronologie alsolue du site. La courche $\mathrm{d}_{2}$, limité an monument au sud et inexistante ou fort peu épaisse au nord, semble être antérieure au premier état en rur de l'édifice. Elle doit être contemporaine d'une construction de bois et de pierres sèches dont nous n'avons rien retrouvé, mais qui affectait en gros la forme du futur sanctuaire. Elle est datable de la seconde moitié du I $^{\text {er }}$ siècle avant J.-t:.

La couche $\mathrm{d}_{1}$, également limitée au monument au sud et au mur médian au nord et à l'ouest, semble être contemporaine du monument maçonné dans son premier état. Elle est datable de la première moitió du ar siècle et relève d'un gallo-romain précoce attardé qu'il n'est pas rare de trouver sur les sites ruraux de Basse Normandie. On remarquera que l'usage du mortier de chaux semble ètre arrivé dans la région arant les autres techniques romaines.

La couche a cet le sol a sont contemporains des amenagements et des embellissements du sanctuaire à la fin du I $^{\mathrm{er}}$ siècle ou au début du $\mathrm{J}^{\mathrm{e}}$ siècle. La formation de la rouche cest interrompue au inilieu du ${ }_{1}$ e siècle et reprend à l'extrême fin du ${ }_{11}{ }^{\mathrm{e}}$ ou au début du ${ }^{\mathrm{e}}$. Enfin, la couche cest surmonté d'une couche de remblai b qui se forme après l'abandon ou la destruction du sanctuaire au milieu du ree.

Le sanctuaire de Baron témoigne d'un fort particularisme indigène rural, puisque, dans lir couche d datable du gallo-romain précoce, on ne trouve que de la céramique et du mobilier de tradition laténienne; à la mème époque, la romanisation des villes est plus poussée. car à Vieux, par exemple, des sondages ont montré que l'on trouvait de la céramique sigillée dès le début du zer siècle. Quant à la lacune dans l'ocrupation de Baron entre le II $^{\mathrm{e}}$ et le re sière (lacune partielle, car nous avons la preuve que les dépendances du sanctuaire ont été orcupées pendant rette période), elle s'explique par la prospérité de Vieux pendant cette époque. Le sanctuaire indigène a sans doute été abandonné au profit d'un sanctuaire officiel nouvellement ronstruit, puis il a eté réorcupe au we siècle après la destruction de Vieux, lors de lat réapparition des traditions celtiques.

Qurls ont été les aspects successifs du sanctuaire? A La Tène finale, entre j0 et 1, ce devait ettre un monument en bois de forme ovale, ayant en gros les dimensions du futur monument gallo-romain. Il etait peut-ètre constitué d'un simple péribole en matériaux légers, et, s'il y arait des galeries, elles étaient en bois et couvertes en chaume.

A l'époque du gallo-romain précoce, entre 1 et 50 environ, l'édifice est reconstruit avec des fondations en dur. A cet etat correspond la construction du mur de galerie médian, du mur intérieur sur tout le pourtour du monument, du mur extérieur au sud seulement. Le sanctuaire possède alors une galcric intérieure complète, at un trongon de galerie extérieure. Less galeries comportent sans doute des colonnes de bois sur les faces interne et externe; le mur central, plus solide, est soit à colonnade, soit plutôt plein et il supporte la toiture de tuiles des galeries. Au début du $\mathrm{II}^{\mathrm{e}}$ siècle, la galerie extérieure. inutile et inachevée, est condamnée. Comme désormais elle ne sert plus à la déambulation, elle est obstruée par une petite salle, et on arlosse en même trmps trois petites salles au mur extérieur. De plus, certaines parties du sanctuaire recoivent alor's une ornementation sculptée. Du milieu du ${ }_{I}{ }^{e}$ siècle à la lin du III $^{\mathrm{e}}$ ou au début du $\mathrm{IV}^{\mathrm{e}}$, le sanctuaire est abandonné et s'effondre par vétusté. Au début du we siècle, il est réoccupé et sommairement reconstruit. 

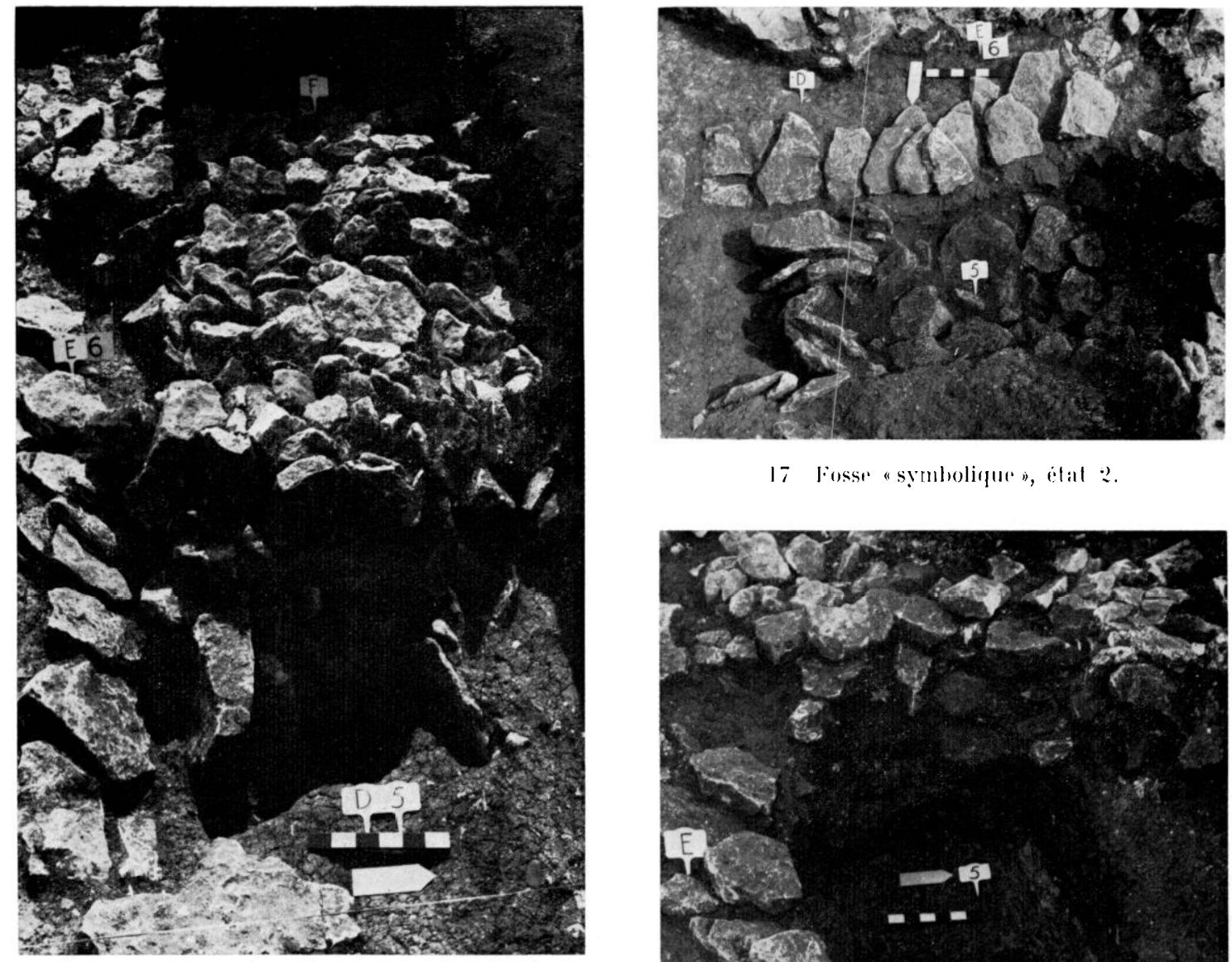

17 Fusse "symboliquu ", ital :

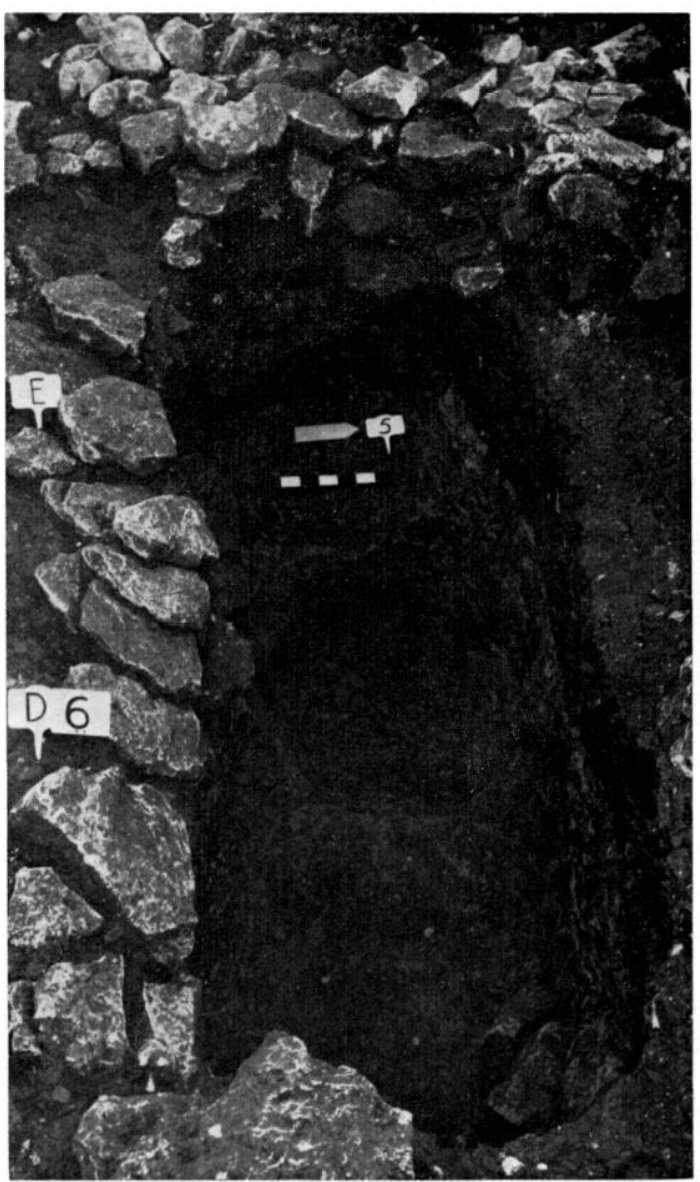

Des foulles annexes, pratiquees a $100 \mathrm{~m}$ du monument et lans laaxe le lientrex. ont. peut-ètre livé les clements les plus originaux et les plus intéressants du sile, a savoir une "fosse symbolique " et un habitat pré-romain.

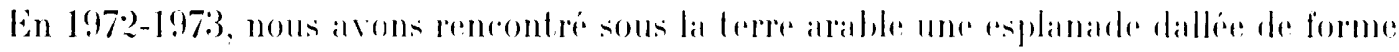

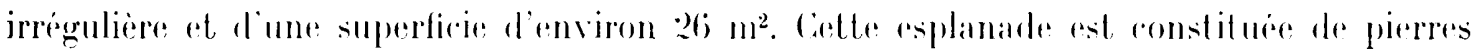

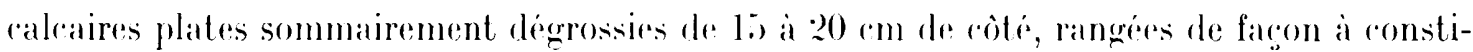


tuer un dallage grossier épais de 0,25 m. Partant de l'hỵpothese que ce dallage n avait pats été établi en vain, nous l'avons traversé par deux tranchées. A peu pres au centre de l'esplanarle, au point le plus haut, nous avons rencontre sous le dallage des structures verticales qui se sont révélées appartenir à une fosse remplie de pierres. Sous les trois ranges de pierres calcaires posées à l'horizontale du dallage, des pierres à chant sont apparues. les bords est et sud de la fosse sont alors soulignes par des pierres calcaires inclinées seton un pendage de $26^{\circ}$ en moyenne (fig. 16). On a rencontre ensuite dans la fosse ring remplissages sureessifs de dalles à plat (fig. 17). Cne fois la fosse vidée, des pierres demeurent dans une cavité centrale, qui a l'aspect d'une curette parfaitement circulaire de $0.60 \mathrm{~m}$ de diametre et de $0,30 \mathrm{~m}$ de profondeur fig. 18). Cette fosse a éte creusée dans les courhes archeologiques gallo-romaines, pré-romaines, et dans la roche calcaire naturelle. lille a $1,90 \mathrm{~m}$ de long sur 0,75 $\mathrm{m}$ de large. Sa profondeur varie de $1 \mathrm{~m}$ à l'ouest. à $1.20 \mathrm{~m}$ à l'est et à $1,50 \mathrm{~m}$ dans lia cavité centrale (fig. 19). Elle ne contient ni ossements ni mobilier, a l'exception d'une perle. en rerre qui s'est infiltrée. Comme il est exclu qu'clle ait été violée, on peut la ronsiderer comme une sépulture symbolique. Les pierres dresséses à la tête de la fosse auraient servi de stèle et la sépulture aurait eu une orientation est-ouest. La curette centrale ligure peut-être le puits miniature permettant le passage entre le monde des morts at celui des vivants; peut-être est-e un monument, commémoratif d'un érénement qui aurait reu lieu dans le sanctuaire; peut-être est-ce une sorte de "monument aux morts". Mais de là a voir dans le sanctuaire de Baron un "sancluaire funeraire" eomme le fit A. Cirenier pour Sanxay (Vienne), Chassenon (Charente), Ie Jonon (Vosges), ou Saint-Ilerd-les-()ussines (Correzze), il y a un pas que nous no franchirons pas, car il n'y a jamais cul de véribable sépulture à còté d'un sanctuaire gallo-romain. Le pseudo-monument funeraire de Baron est postérieur au $\mathrm{Il}^{\mathrm{e}}$ siecle, mais nous sommes frappé par son caratetere archaïgue, car un tel monument

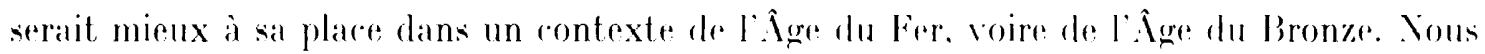
pensons être en présence d'une résurgence d'une tradition indigène, et celte hy potheses séclaire à la lumière des déecouvertes dont nous allons parler maintenant.

En effet, par un hasard de la fouille, nous avons découvert un habitat pré-romain a proximité de cette structure. La stratigraphie du site dans re secteur ast proche de celle que l'on a observée sur le monument. La couche pré-romaine d, l'argile noire, a live de nombreuses traces d'habitat : fragments d'argile de clayonnage avec traces de branchages. foyer au-rlessus de la roche ralcaire naturelle, reposant sur un lit de pierres calcaires. constitué d'une croùte d'argile cuite et entouré d'un abondant mobilier métallique et céramique (fig. 20), fosses irrégulières, profondes de 0,30 m, reusées dans la roche callaire, sans doute aménagées par les constructeurs de la cabane. mais dont la destination demeure hypothetique. Trop grandes pour etre des trous de poteaux, sans rapport avec la fosse

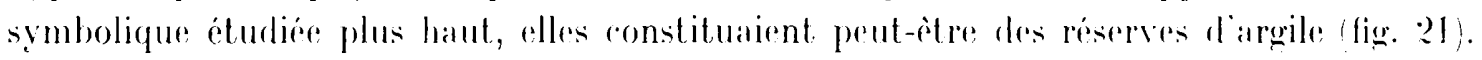

Du point de vue du mobilier, on a recueilli environ 400 tesons d'une céramique dans l'ensemble plus fine et mieux finie que celle qui a etr trouvée sur le monument. Les décors sont nombreux et surtout représentés par des cherons ou des quadrillages faits au bru-

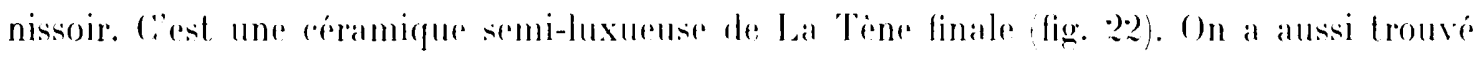
une monnate gauloise tardive, analogure à celles qui ont dete recueillies dans le monument. 

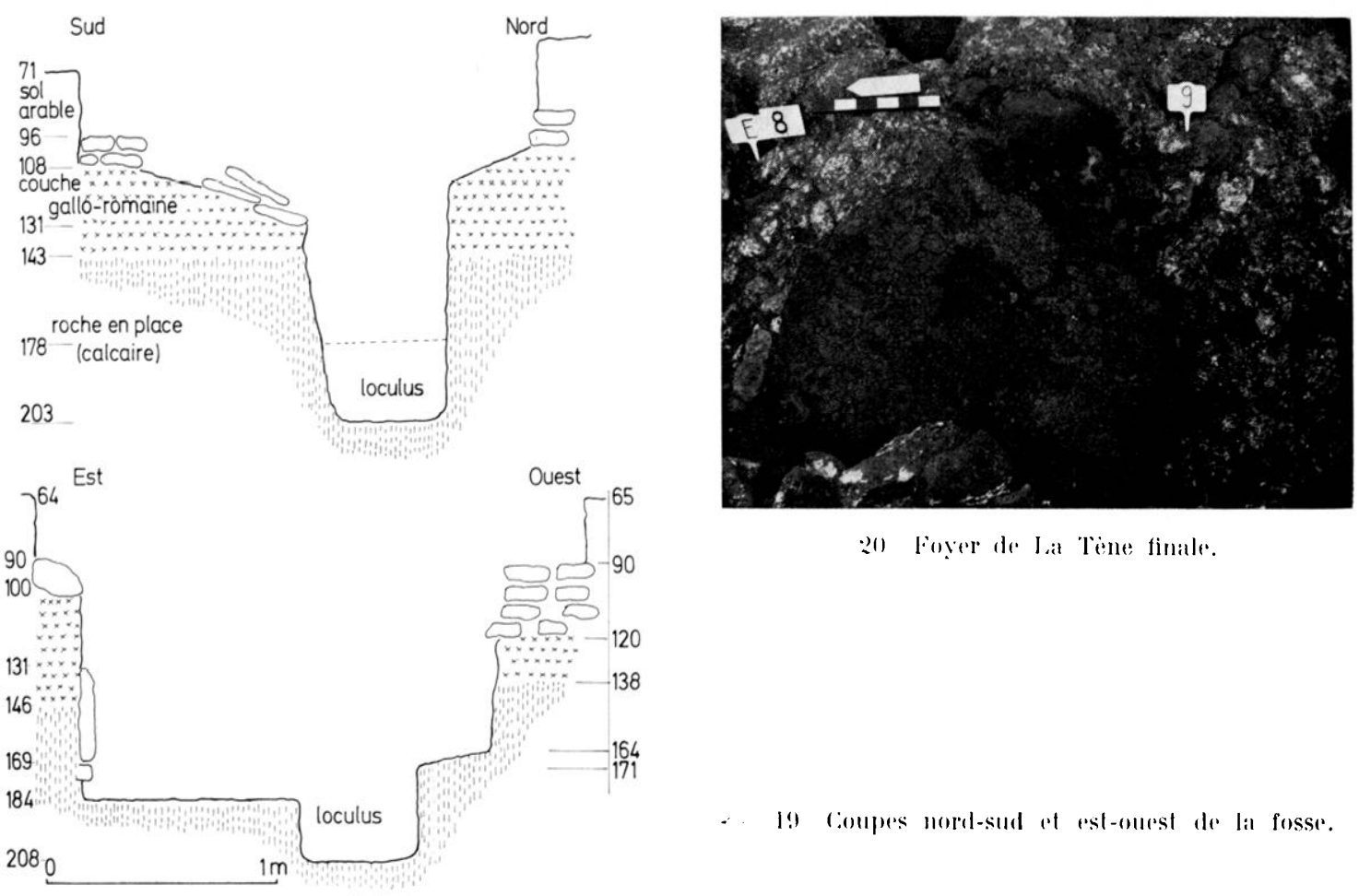

211 Foyer de Ja Tène finale.

- I! Coupes nord-sud of est-ouest de la fosse.
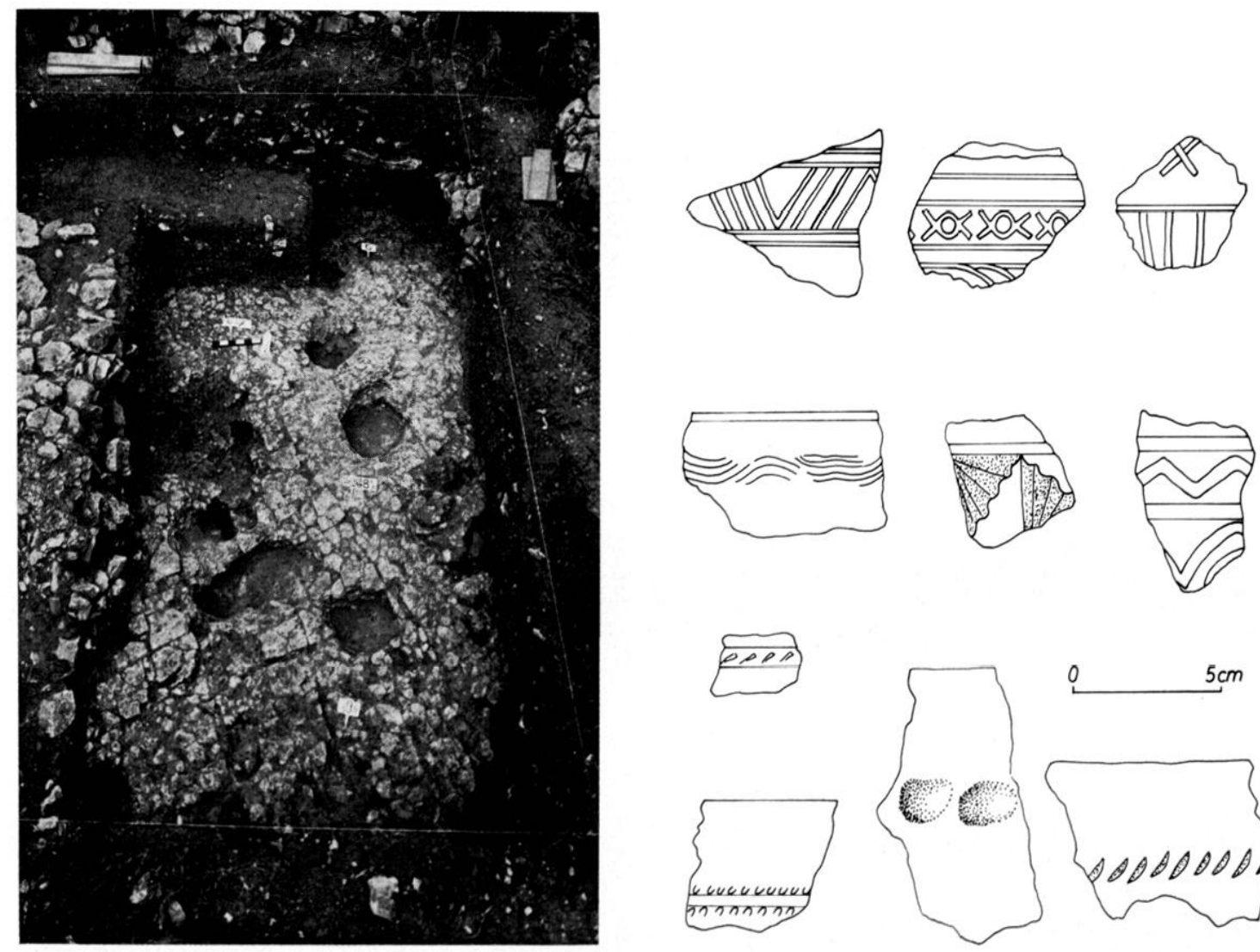

:I Fosses de la Tene finale dans la planuelle calcaire naturelle.

:2: ciramigue decoree de la Téne finale. 
quelques anneaux et une boucle de ceinture en bronze, deux lames de fer spiralés, un couteau, une douille de lance, des anneaux en fer, enfin une meule.

Ces dernieres découvertes, mobilier et structures de la T'ene finale, permettent daffirmer que le site de Baron étail occupé sous l'Indépendance ou du moins vers jo arant J.-l. Les sondages que nous avons pratiqués à Vieux n'ayant pas livré de niveaul gaulois jusqu’à present, il est possible que le site du llesnil de Baron, dont nous n'arons pu fouiller encore qu'une petite parlie, ait eté l'un des principaux habitats des Viducasses avant la Conquête, et que Vieux, chefflieu de cite gallo-romain, ne soit, en dépit de son nom gaulois Aregenue, qu'une creation romaine.

Dominique: Br:kTs: 